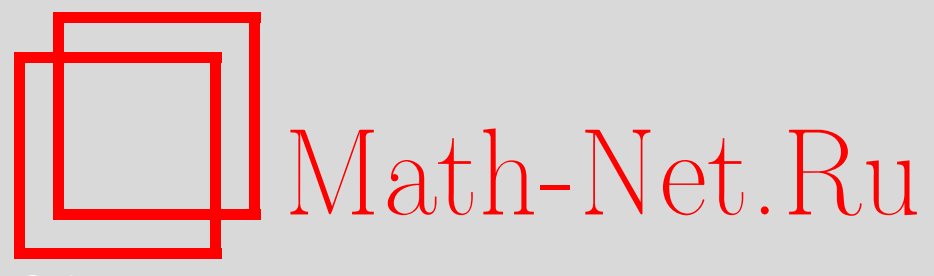

Д. О. Синицын, Асимптотическая гамильтонова редукция для геодезических на деформированных сферах и преобразование Функа-Минковского, Матем. заметки, 2011, том 90, выпуск 3, 474-477

DOI: https://doi.org/10.4213/mzm9227

Использование Общероссийского математического портала Math-Net.Ru подразумевает, что вы прочитали и согласны с пользовательским соглашением http://www . mathnet.ru/rus/agreement

Параметры загрузки:

IP: 54.147 .182 .235

26 апреля 2023 г., 10:47:27

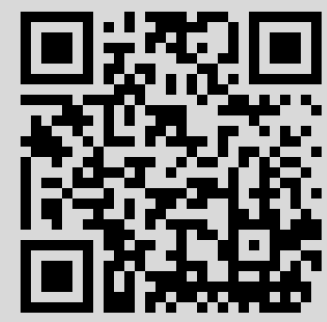




\section{Асимптотическая гамильтонова редукция для геодезических на деформированных сферах и преобразование Функа-Минковского}

\section{Д. О. Синицын}

В задачах нахождения геодезических линий на двумерных поверхностях точные аналитические решения известны только для ограниченнго круга специальных случаев [1]. Поэтому представляется разумным применение для этих задач асимптотических методов аналитической механики.

Рассматриваются геодезические на поверхностях, полученных малой деформацией стандартной двумерной сферы. С помощью осреднения производится асимптотическая редукция уравнений геодезических к гамильтоновой системе с одной степенью свободы. Для специального класса деформаций сферы указанная редукция была применена ранее в [2]. В настоящей работе производится редукция для произвольной гладкой малой деформации сферы и доказывается, что осредненная система имеет гамильтониан, получаемый из функции, задающей деформацию сферы, с помощью интегрального преобразования Функа-Минковского (известного также как преобразование Радона для группы $S O(3)$ ), см. [3]. Функция, в главной части совпадающая с осредненным гамильтонианом, использовалась Пуанкаре для изучения замкнутых геодезических [4].

Рассмотрим движение частицы единичной массы по поверхности

$$
\varphi(\vec{x})=0, \quad \text { где } \quad \varphi(\vec{x})=\sum_{i=1}^{3} x_{i}^{2}-1+\varepsilon \psi(\vec{x}), \quad \varepsilon \ll 1,
$$

т.е. поверхность получена малым возмущением $\psi(\vec{x})$ стандартной сферы. Движение частицы описывается уравнением Лагранжа первого рода:

$$
\ddot{\vec{x}}=\lambda \frac{\partial \varphi}{\partial \vec{x}} .
$$

Ключевое наблюдение состоит в том, что траектория частицы на малом интервале времени проходит вблизи большого круга (точного решения невозмущенной задачи - геодезической на сфере). На больших интервалах расположение этого большого круга претерпевает эволюцию со временем. Расположение большого круга может быть описано вектором нормали к его плоскости, в качестве которого служит угловой момент частицы:

$$
\vec{L}=\vec{x} \times \dot{\vec{x}} .
$$

Его производная по времени

$$
\dot{\vec{L}}=\vec{x} \times \ddot{\vec{x}}=\vec{x} \times \lambda \frac{\partial \varphi}{\partial \vec{x}}=\lambda \vec{x} \times\left(\vec{x}+\varepsilon \frac{\partial \psi}{\partial \vec{x}}\right)=\lambda \varepsilon \vec{x} \times \frac{\partial \psi}{\partial \vec{x}} .
$$

Мы удерживаем в этом уравнении только члены первого порядка по $\varepsilon$. Ввиду этого заменяем $\lambda$ на его главную часть нулевого порядка $\lambda_{0}=-\dot{\vec{x}}^{2}=$ const (кинетическая энергия сохраняется). Рассмотрим траектории в натуральной параметризации, для которых $\dot{\vec{x}}^{2}=1$, а значит, $\lambda_{0}=-1$ и

$$
\dot{\vec{L}}=-\varepsilon \vec{x} \times \frac{\partial \psi}{\partial \vec{x}} .
$$

Работа выполнена при поддержке Российского фонда фундаментальных исследований (гранты №o 10-01-00748-a, 11-02-01462-a), программы "Ведущие научные школы" (грант № НШ3224.2010.1), АВЦП "Развитие научного потенциала высшей школы” (проект № РНП-2.1.1.3704), ФЦП "Научные и научно-педагогические кадры инновационной России" на 2009-2013 гг. (номера госконтрактов: 14.740.11.0794, 02.740.11.5213). 


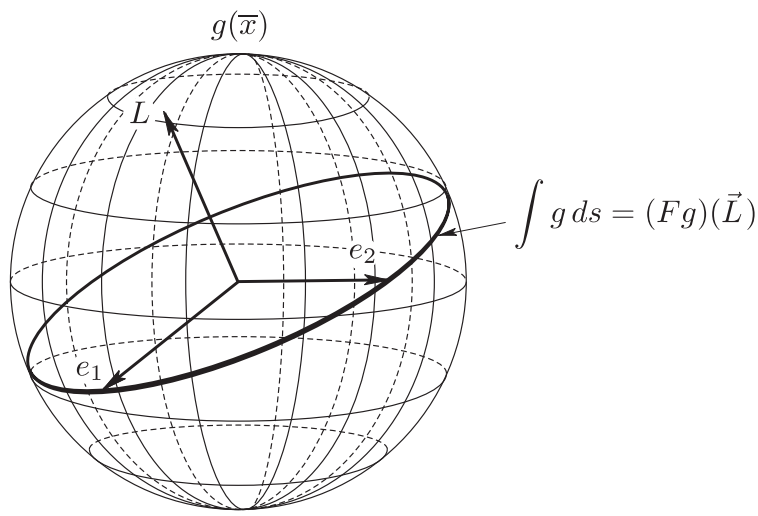

Рис. 1. Преобразование Функа-Минковского: интегралы функции на сфере по большим кругам

Компоненты углового момента - медленные переменные, фаза обращения по большому кругу - быстрая переменная. Таким образом, эволюция момента может быть асимптотически описана с помощью метода осреднения, [5]. Рассмотрим точное решение невозмущенной системы с заданным $\vec{L}$ :

$$
\vec{x}_{\vec{L}}(t)=\cos t \vec{e}_{1}(\vec{L})+\sin t \vec{e}_{2}(\vec{L}),
$$

где векторы $\vec{e}_{1}(\vec{L}), \vec{e}_{2}(\vec{L})$ образуют ортонормированный базис вместе с вектором $\vec{e}_{3}(\vec{L})=$ $\vec{L} / L$, см. рис. 1 .

Осреднение функции на сфере по периоду этого решения эквивалентно применению интегрального преобразования $F$ Функа-Минковского, сопоставляющего функции на сфере ее интегралы по большим кругам, [3]:

$$
(F g(\vec{x}))(\vec{L})=\int_{0}^{2 \pi} g\left(\cos t \vec{e}_{1}(\vec{L})+\sin t \vec{e}_{2}(\vec{L})\right) d t .
$$

Теорема 1. Осреднение уравнений (1) динамики момента по периоду точного решения (2) невозмущенной задачи дает гамильтонову систему

$$
\dot{\vec{L}}=\{\vec{L}, H(\vec{L})\}
$$

где скобки Пуассона имеют вид

$$
\left\{L_{i}, L_{j}\right\}=\sum_{k=1}^{3} \varepsilon_{i j k} L_{k}
$$

(здесъ $\varepsilon_{i j k}$ - символ Леви-Чивить), а гамильтониан равен (с точностью до числового множителя) преобразованию Функа-Минковского, примененному к функции вұ, задающей деформачию сферы:

$$
H(\vec{L})=\frac{1}{2 \pi} F(\varepsilon \psi) .
$$

ДокАзАтельство. Осредняя (1), имеем

$$
\begin{aligned}
\dot{\vec{L}} & =-\frac{1}{2 \pi} \varepsilon \int_{0}^{2 \pi} \vec{x}_{\vec{L}}(t) \times \frac{\partial \psi}{\partial \vec{x}}\left(\vec{x}_{\vec{L}}(t)\right) d t \\
& =-\frac{1}{2 \pi} \varepsilon\left(F\left(\vec{x} \times \frac{\partial \psi}{\partial \vec{x}}\right)\right)(\vec{L})=-\frac{1}{2 \pi} \varepsilon(F \circ \vec{l} \psi)(\vec{L}),
\end{aligned}
$$


где обозначен оператор $\vec{l}=\vec{x} \times \partial / \partial \vec{x}$ (здесь $\vec{x}-$ векторный аргумент функции, к которой применяется оператор). Справедлива следующая основная

Лемма 1. Имеет место равенство

$$
F \circ \vec{l}=\vec{l} \circ F .
$$

ДокАзАтЕльство. Для ее доказательства используем следующее

Свойство 1. Преобразование $F$ коммутирует с трехмерными вращениями: для $R \in$ SO(3) выполнено

$$
R \circ F=F \circ R, \quad \text { где } \quad R \psi(\vec{x})=\psi\left(R^{-1} \vec{x}\right) .
$$

Действительно,

$$
(R(F \psi))(\vec{L})=\int_{0}^{2 \pi} \psi\left(\vec{x}_{R^{-1} \vec{L}}(t)\right) d t=\int_{0}^{2 \pi} \psi\left(R^{-1} \vec{x}_{\vec{L}}(t)\right) d t=(F R \psi)(\vec{L}) .
$$

Заметим, что компоненты $\vec{l}$ суть операторы бесконечно малых поворотов:

$$
l_{i}=-\left.\left(\frac{d}{d \alpha} R_{i}(\alpha)\right)\right|_{\alpha=0},
$$

где $R_{i}(\alpha)$ - вращение вокруг оси $i$ на угол $\alpha$. Поэтому

$$
\left(l_{i} \circ F\right) \psi=-\left.\frac{d}{d \alpha}\left(\left(R_{i}(\alpha) \circ F\right) \psi\right)\right|_{\alpha=0}=-\left.\frac{d}{d \alpha}\left(\left(F \circ R_{i}(\alpha)\right) \psi\right)\right|_{\alpha=0}=\left(F \circ l_{i}\right) \psi .
$$

Пользуясь леммой, мы можем поменять местами операторы в (4):

$$
\dot{\vec{L}}=-\frac{1}{2 \pi} \varepsilon(\vec{l} \circ F \psi)=-\frac{1}{2 \pi} \varepsilon\left(\vec{L} \times \frac{\partial}{\partial \vec{L}}\right)(F \psi)(\vec{L}) .
$$

Но легко видеть, что оператор $\vec{L} \times \partial / \partial \vec{L}$ действует на произвольную функцию $G(\vec{L})$ как взятие ее скобки Пуассона с $\vec{L}: \vec{L} \times \partial G / \partial \vec{L}=\{G, \vec{L}\}$ для базисных скобок (3). Поэтому осредненная система имеет гамильтонову форму:

$$
\dot{\vec{L}}=\{\vec{L}, H(\vec{L})\}, \quad \text { где } \quad H(\vec{L})=\frac{1}{2 \pi} F(\varepsilon \psi) .
$$

Теорема доказана.

Алгебра Пуассона (3) имеет функцию Казимира $\vec{L}^{2}$. Поэтому фазовое пространство осредненной системы - двумерная сфера $\vec{L}^{2}=$ const. Таким образом, мы имеем гамильтонову систему с одной степенью свободы, которая, следовательно, интегрируема. Топологическая структура ее динамики описывается фазовым портретом на двумерной сфере. Траектории суть линии уровня гамильтониана $H(\vec{L})=$ const. Стационарные точки системы соответствуют геодезическим, лежащим в окрестности плоского сечения поверхности.

Вопросы осреднения в системах с одной быстрой фазой рассматривались в [6], см. также [7]. Из описанной Нейштадтом [6] возможности проведения многократного осреднения (некоторые уточнения см. в [8]) следует, что для аналитических функций деформации $\psi(\vec{x})$ векторное поле, задающее исходный геодезический поток, экспоненциально близко к некоторому гамильтонову векторному полю, задающему вполне интегрируемую систему. Экспоненциальная близость здесь понимается в том смысле, что разность указанных векторных полей имеет порядок $O\left(e^{-\alpha / \varepsilon}\right), \alpha>0$. Постоянную $\alpha$ в принципе можно оценить, используя методы, изложенные в [8], см. также [9].

Кроме того, к данной системе применима теория КАМ для систем с собственным вырождением с двумя степенями свободы [10]. По теореме об отсутствии эволюции для таких 
систем при возмущении, снимающем вырождение, получаем, что если для данной функции деформации сферы $\psi(\vec{x})$ условие снятия вырождения выполнено, то на бесконечном интервале времени действие $\vec{L}^{2}$, сопряженное быстрой фазе, и действие осредненной системы, которое функционально связано с ее энергией $H(\vec{L})$, остаются $\varepsilon$-близки к своим начальным значениям. Поэтому траектории момента $\vec{L}$ в точной системе проходят в $\varepsilon$-окрестности совместных линий уровня $\vec{L}^{2}=$ const, $H(\vec{L})=$ const, являющихся траекториями осредненной системы.

В целом данная редукция позволяет исследовать общую структуру расположения геодезических на деформированной сфере.

Автор благодарит В. Л. Голо за внимание к этой работе.

Автор признателен С. Ю. Доброхотову, А. И. Шафаревичу, А. И. Нейштадту, Д. В. Трещеву за полезные замечания.

\section{СПИСОК ЦИТИРОВАННОЙ ЛИТЕРАТУРЫ}

[1] Б. А. Дубровин, С. П. Новиков, А. Т. Фоменко, Современная геометрия. Методъ и приложения, т. 1-3, УРCC, M., 2001. [2] V. L. Golo, D. O. Sinitsyn, Писъма в ЭЧАЯ, 5:3 (2008), 473-478. [3] И. М. Гельфанд, С. Г. Гиндикин, М. И. Граев, Избранные задачи интегральной геометрии, Добросвет, М., 2010. [4] H. Poincaré, Trans. Amer. Math. Soc., 6:3 (1905), 237-274. [5] В. И. Арнольд, Математические методы классической механижu, УРСС, М., 2003. [6] А.И. Нейштадт, ПММ, 48:2 (1984), 197-204. [7] J. Brüning, S. Yu. Dobrokhotov, K. V. Pankrashkin, Russ. J. Math. Phys., 9:1 (2002), 14-49; 9:4 (2002), 400-416. [8] Д. В. Трещев, Рег. хаот. дин., 2:3-4 (1997), 9-20. [9] Д. В. Трещев, Введение в теорию возмущений гамильтоновых систем, Библиотека студента-математика, 6, Фазис, М., 1998. [10] В. И. Арнольд, В.В.Козлов, А. И. Нейштадт, "Математические аспекты классической и небесной механики", Динамические системы - 3, Итоги науки и техн. Сер. Соврем. пробл. мат. Фундам. направления, 3, ВИНИТИ, М., 1985, 5-290.

Д. О. Синицын

Московский государственный университет им. М. В. Ломоносова

E-mail: d_sinitsyn@mail.ru
Поступило 10.03.2011 